\title{
Supplemental application of phosphorus improves yield, quality and net returns of Gossypium hirsutum
}

\author{
Ayaz Ahmad ${ }^{1 *}$, Hakoomat Ali $^{1}$, Shabir Hussain ${ }^{1}$, Waseem Hassan ${ }^{1}$ \\ and Riaz Ahmad ${ }^{2}$ \\ 1. Department of Agronomy, Bahauddin Zakariya University, 60800 Multan-Pakistan \\ 2. Department of Horticulture, Bahauddin Zakariya University, 60800 Multan-Pakistan \\ *Corresponding author's email: ayazagronomist@gmail.com \\ Citation
}

Ayaz Ahmad, Hakoomat Ali, Shabir Hussain, Waseem Hassan and Riaz Ahmad. Supplemental application of phosphorus improves yield, quality and net returns of Gossypium hirsutum. Pure and Applied Biology. Vol. 9 , Issue 4, pp2577-2588. http://dx.doi.org/10.19045/bspab.2020.90274

\begin{tabular}{|c|c|c|c|}
\hline Received: 09/04/2020 & Revised: 01/07/2020 & Accepted: 10/07/2020 & Online First: 06/08/2020 \\
\hline
\end{tabular}

\section{Abstract}

Cotton is a white gold due to its high economic values. Phosphorus is an important constituent that is essential for seed development and yield. Efficient use of phosphorus plays a significant role to achieve quality production in form of yield to overcome the problems. A field study comprised of four phosphorus levels viz. $\left(\mathrm{P}_{0}=0 \mathrm{kgha}^{-1}, \mathrm{P}_{1}=50 \mathrm{kgha}^{-1}, \mathrm{P}_{2}=100 \mathrm{~kg}\right.$ $\mathrm{ha}^{-1} \mathrm{P}_{3}=150 \mathrm{~kg} \mathrm{ha}^{-1}$ ) and cultivars viz CIM-598, MNH-886, CIM-616, CIM-612, FHLALAZAR during 2015 and 2016. Application of phosphorous significantly improved quality and yield in terms of plant height $(161.33 \mathrm{~cm})$, bolls number plant ${ }^{-1}(31.667)$, number of nodes plant ${ }^{-1}(49.000)$, boll weight $(2.200 \mathrm{~g})$, seed cotton harvest $(3481.3 \%)$, seed cotton yield $\left(2928.3 \mathrm{~kg} \mathrm{ha}^{-1}\right)$, seed lint yield $\left(56.667 \mathrm{~kg} \mathrm{ha}^{-1}\right)$, biological yield $\left(0.960 \mathrm{~kg} \mathrm{ha}^{-1}\right)$, and ginning out turn $(0.0227 \%)$. Significant increase was observed for leaf area index $\left(0.980 \mathrm{~m}^{-}\right.$ ${ }^{2}$ ), crop growth rate $\left(1.416 \mathrm{~g} \mathrm{~m}^{-2} \mathrm{day}^{-1}\right)$, net assimilation rate $\left(1.480 \mathrm{~g} \mathrm{~m}^{-2}\right.$ day $\left.^{-1}\right)$, leaf area duration (871.33), phosphorus concentration (0.270), fiber uniformity (70.667), staple length (47.500 mm), fiber strength (33.500 tppsi) and micronair (5.166 $\left.\mu \mathrm{g} \mathrm{inch}^{-1}\right)$. Phosphorus spray of $150 \mathrm{~kg} \mathrm{ha}^{-1}$ is more suitable for greater cotton production.

Keywords: Cotton; Cotton quality; Economic benefits; Fiber uniformity; Phosphorus

\section{Introduction}

Cotton Gossypium hirsutum L. belongs family Malvaceae and comprised of approximately 50 species [1]. Cotton is a unique natural fiber provides medicinal compounds, vegetable oil, organic matter and energy sources to fertile soil, meal and hull for livestock feed and industrial lubricants [2].

Deficiency of phosphorus is major problem in calcareous soils all over the world specially Pakistan [3]. Deficiency of phosphorus decreased in plant growth, photosynthesis and yield [4]. Phosphorus demand has been increased due to the maximum production of cultivars as well as sufficient increase of growth as well as development due to regulation of photosynthesis with rise of $\mathrm{CO}_{2}$ concentrations [3]. However, evaluation of carbon dioxide increased yield, photosynthesis mechanism as well as plant growth. However, phosphorus has a major nutrient for plant response to elevated carbon dioxide $[3,5]$.

Phosphorus deficiency resulting in reduction of dry matter and poor development of cotton roots $[6,7]$. While 
the minimization of phosphorus impact on cotton plants which could be retained $35 \%$ phosphorus in its roots, compared with $14 \%$ in phosphorus sufficient cotton [8]. Moreover, phosphorous utilization occurred maximum in growing season when the root system is fully developed [9].

Phosphorus fertilizer application mainly depends on fertilizer application of phosphorus, type of soil, agronomic implications and geographical site of cultivated crop mainly resulting in improvement of crop productivity $[5, \mathbf{1 0}]$. Different types of genotypes have different efficiency to perform in absorbing fertilizer efficiently due to difference in genetic makeup [11]. The important genetically differences among cotton cultivars can be exploited for breeding efficient cultivars because of fertilizer enhances efficiency [12]. It would preferable to select and identify the important traits between various cotton cultivars that are directly relate to phosphorus efficiency.

Many previous studies have been carried out to investigate the deficiency of phosphate on cotton growth as well as physiological process due to the projected atmospheric carbon dioxide conditions which are limited [8]. So, the maximum root assimilation growth due to stress of phosphorus of cotton could be conflicted on a significant advantage in soluble phosphorus acquisition by cotton. Therefore, suitable cultivars selection according to the prevailing conditions of the region along with important management practices is even more importance for cotton production, although high yield potential is a principal concern.

\section{Materials and methods \\ Experimental details}

Investigate the response of various cotton cultivars to phosphorus application during 2015-2016 (Fig. 1). Eco-meteorological data of experimental site was listed below. Experimental treatments comprises of four phosphorus levels viz. To $=0 \mathrm{kgha}^{-1}, \mathrm{~T}_{1}=$
$50 \mathrm{kgha}^{-1}, \mathrm{~T}_{2}=100 \mathrm{kgha}^{-1} \mathrm{~T}_{3}=150 \mathrm{kgha}^{-1}$ and five cultivars viz. CIM- 598, MNH 886,CIM - 616, CIM- 612, FHLALAZAR. The preparation of seed bed cotton was sown by having row -row distance of $75 \mathrm{~cm}$ as well as plant - plant distance of $25 \mathrm{~cm}$ during 28 May 2015 and 31 May 2016. Urea fertilizers $36 \mathrm{kgha}^{-1}$ applied as per standard role and four Phosphorous levels (0, 50, 100 and 150 $\mathrm{kgha}^{-1)}$, respectively. Randomized complete block design (RCBD) was applied along with three replications. Irrigation as well as crop protection needs were performed as per requirement of the crop. Harvesting was done on 05-11-2015 to 13-11-2016 with the picking interval of 2 weeks.

\section{Observations recorded Agronomic traits}

Plant height was determined from five collected plants through measuring scale. Same plants were used to measure number of bolls per plant. One hundred good open bolls were choose for picking to get 100 boll weights (g) from each treatment. Seed cotton yield was recorded after picking and then weighed to get the yield per hectare $\left(\mathrm{kg} \mathrm{ha}^{-1}\right)$. Ginning out turn $(\%)$ was calculated through weighing cotton lint and cotton seed. According to previous studies $[13,14]$.

\section{Allometry traits}

Leaf area index was measured by dividing leaf area over the land area. Crop growth rate was measured by collected weighed samples of 20,40, $50 \mathrm{~g}$ of green leaves, stalks and bolls which were gained from different stages of crop growth and were dried in the oven at $80 \mathrm{C}^{0}$. Hunt [20] gave the formula to calculate net assimilation rate.

\section{Statistical analysis}

The statistical software, Statistix 8.1 (Tallahassee Florida, USA) was used to examine two years collected data. Due to two factors; cultivars and treatment threeway analysis of variance were applied. Year interaction was non-significant and did not described. Means were separated 
through LSD test at 5\% level of probability.

\section{Results}

Descriptive statistics for individual effect of cultivars, phosphorus levels and their interactive effects was presented in the (Table 1). Regarding the individual effect of cultivars, larger plant height was calculated in cultivars MNH-886 and CIM-616, while smaller plant height was measured in cultivars CIM-616 and FHLalazar. Number of bolls plant ${ }^{-1}$ were higher in cv. CIM-616, while lower was in CIM-598 and FH-Lalazar. CIM-616 had the maximum number of nodes plant ${ }^{-1}$ and boll weight as compared to all other studied traits. The performance of all the studied cultivars was similar for the seed cotton yield and seed cotton harvest. The highest lint yield was measured in MNH886, while lowest was in CIM-616. CIM612 had the highest biological yield, followed by CIM-616 than all the studied cultivars. FH-Lalazar, followed by CIM616 and CIM-598 had the highest ginning out turn as compared to other cultivars (Table 2). CIM-616 had the highest leaf area index, crop growth rate and micronair, while the lowest leaf area index, crop growth rate, net assimilation rate and leaf area duration was in $\mathrm{FH}$ Lalazar (Table 3). MNH-886 had the maximum net assimilation rate, leaf area duration, phosphorus concentration, fiber uniformity, staple length and fiber strength (Table 3).

Regarding the effect of phosphorus on agronomic and yield related traits, phosphorus concentration (150 kg ha-1) showed the better results as compared to other concentrations. The highest plant height, number of boll plant ${ }^{-1}$, number of nodes plant $^{-1}$, boll weight, seed cotton yield, lint yield, biological yield, seed cotton harvest and ginning out turn were recorded during phosphorus application as $150 \mathrm{~kg} \mathrm{ha}^{-1}$ than other application doses (Table 4). Individual effect of phosphorus on physiological and fiber quality traits were presented (Table 5). Phosphorus application $\left(150 \mathrm{~kg} \mathrm{ha}^{-1}\right)$ significantly enhanced leaf area index, crop growth rate, net assimilation rate, leaf area duration, phosphorus concentration, fiber uniformity, staple length, fiber strength and micronair (Table 5).

As regards, the interaction of cultivars and phosphorus effect on agronomic and yield related traits, the highest plant height was recorded during $\left(\mathrm{P}_{3} \times \mathrm{V}_{2}\right)$ and $\left(\mathrm{P}_{3} \times \mathrm{V}_{3}\right)$, while the lowest plant height was measured during $\left(\mathrm{P}_{0} \times \mathrm{V}_{4}\right)$ and $\left(\mathrm{P}_{0} \times \mathrm{V}_{5}\right)$ as compared to other genotypes. The maximum number of bolls plant ${ }^{-1}$ was counted in $\left(\mathrm{P}_{3} \times \mathrm{V}_{3}\right)$, while the minimum number of bolls plant ${ }^{-1}$ was calculated in $\left(\mathrm{P}_{0} \times \mathrm{V}_{5}\right)$ and $\left(\mathrm{P}_{0} \times \mathrm{V}_{1}\right)$. Number of nodes plant $^{-1}$ were not significantly varied in the current studied. Boll weight was higher in $\left(\mathrm{P}_{3} \times \mathrm{V}_{1}\right),\left(\mathrm{P}_{3} \times \mathrm{V}_{2}\right),\left(\mathrm{P}_{0} \times \mathrm{V}_{5}\right)$ and $\left(\mathrm{P}_{3} \times\right.$ $\left.\mathrm{V}_{5}\right)$, while lower boll weight was in $\left(\mathrm{P}_{0} \times\right.$ $\left.\mathrm{V}_{3}\right)$, followed by $\left(\mathrm{P}_{0} \times \mathrm{V}_{1}\right),\left(\mathrm{P}_{0} \times \mathrm{V}_{2}\right),\left(\mathrm{P}_{1}\right.$ $\left.\times V_{3}\right)$ and $\left(P_{0} \times V_{4}\right)$. The maximum seed cotton yield was in $\left(\mathrm{P}_{3} \times \mathrm{V}_{2}\right)$ and $\left(\mathrm{P}_{3} \times\right.$ $\mathrm{V}_{4}$ ), while the minimum was measured in $\left(\mathrm{P}_{0} \times \mathrm{V}_{4}\right)$. Lint yield was higher in $\left(\mathrm{P}_{3} \times\right.$ $\left.V_{2}\right)$, followed by $\left(P_{3} \times V_{4}\right)$. However, the lowest was measured in $\left(\mathrm{P}_{0} \times \mathrm{V}_{5}\right)$ and $\left(\mathrm{P}_{1}\right.$ $\left.\times V_{5}\right)$. The highest biological yield was measured in $\left(\mathrm{P}_{3} \times \mathrm{V}_{4}\right)$, followed by $\left(\mathrm{P}_{2} \times\right.$ $\left.\mathrm{V}_{4}\right)$, while the lowest was calculated in $\left(\mathrm{P}_{0}\right.$ $\left.\times \quad V_{2}\right)$ and $\left(\mathrm{P}_{1} \times \mathrm{V}_{2}\right)$. Phosphorus application and cultivars were not showed significant effect on ginning out turn (Table 6). Effect of phosphorus on physiological and fiber quality traits of cotton cultivars were presented (Table 7). The maximum leaf area index was measured in $\left(\mathrm{P}_{2} \times \mathrm{V}_{3}\right)$, while the minimum leaf area index was measured in $\left(\mathrm{P}_{0} \times \mathrm{V}_{1}\right)$, followed by $\left(\mathrm{P}_{3} \times \mathrm{V}_{5}\right)$. Crop growth rate and net assimilation rate were significantly higher in $\left(\mathrm{P}_{3} \times \mathrm{V}_{4}\right)$, while lower were measured in $\left(\mathrm{P}_{0} \times \mathrm{V}_{1}\right)$. The highest leaf area duration was measured in $\left(\mathrm{P}_{3} \times \mathrm{V}_{2}\right)$, while the lowest was calculated in $\left(\mathrm{P}_{0} \times\right.$ $\left.V_{5}\right)$. The highest phosphorus concentration was calculated $\left(\mathrm{P}_{1} \times \mathrm{V}_{3}\right)$ and $\left(\mathrm{P}_{3} \times \mathrm{V}_{5}\right)$, followed by $\left(\mathrm{P}_{0} \times \mathrm{V}_{3}\right),\left(\mathrm{P}_{2} \times \mathrm{V}_{3}\right),\left(\mathrm{P}_{3} \times\right.$ $\left.V_{3}\right),\left(P_{2} \times V_{2}\right)$ and $\left(P_{2} \times V_{5}\right)$. However, the 
lowest phosphorus concentration was measured in $\left(\mathrm{P}_{2} \times \mathrm{V}_{3}\right)$. Fiber quality traits i.e. fiber uniformity, staple length and fiber strength were significantly higher in $\left(\mathrm{P}_{3} \times\right.$ $\mathrm{V}_{2}$ ). The highest micronair was recorded in $\left(\mathrm{P}_{3} \times \mathrm{V}_{3}\right)$ than all other interactions of the current study (Table 7). Detailed description of cost benefit ratio was evaluated as presented in the (Table 8).

\section{Discussion}

Plant height was the highest in MNH-886 and CIM-616 treated with phosphorus 150 $\mathrm{kgha}^{-1}\left(\mathrm{~T}_{3}\right)$, while the lowest was in CIM612, followed by FH-LALAZAR as control $\left(\mathrm{T}_{0}\right)$. Current work is accordance to previous results because these exhibited a positive reaction of phosphorus supplement in cotton crop [15]. Number of bolls per plant is an anticipated trait that is involved in seed cotton yield. Spray of phosphorus concentration (150 kg ha-1) significantly improved the number of bolls per plant than controlled treatment. Among micronutrients i.e. phosphorus is considered to be important for cell enlargement as well as for meristematic tissue development. Furthermore, phosphorus is causing an encouraging effect on flower buds as well as plant bolls. These consequences are in line with earlier consequences as described $[15,16]$. Number of nodes per plant was the greatest in cultivar CIM- 616 treated with phosphorus $150 \mathrm{kgha}^{-1}\left(\mathrm{~T}_{3}\right)$ and the minimum numbers of nodes were in four cultivars such as CIM-612, CIM- 598, MNH- 886 and FH-LALAZAR without any treatment of phosphorus $\left(\mathrm{T}_{0}\right)$. These results correlated with [17]. Micronutrients especially phosphorus is also meaningfully increased boll weight than controlled treatment, as treatment rate was improved up to $150 \mathrm{~kg} \mathrm{ha}^{-1}$ [18]. However, photosynthetic balance as well as stomatal regulation was limited. However, carbon dioxide was found to be decreased when phosphorus deficiency occurs. Present results are in accordance with the findings of previous studies as obtained by Sawan et al. [19]. Phosphorus is macronutrient significantly involved in stimulation of flowering of cotton cultivars. Therefore, current study indicated that increase of flowering and boll development provides higher crop yield to farmers. Earlier results confirmed that phosphorus had significantly enhances seed cotton yield [16, 20]. Furthermore, present consequences were also agreed by earlier research [21]. Lint and biological yield as well as seed cotton harvest was the maximum from the highest concentration applied (150 kg ha-1). However, current findings were in accordance as stated Sawan et al. [19] because good nutrient response as well as their ease of use resulting in initiation as well as formation of higher number of fibers per seed. Ginning out turn was enhanced due to higher phosphorus concentration. Environmental factors, different biotic and abiotic stresses and genetic make-up are major cause of variation in production of different agronomic crops [2].

Leaf area index and crop growth rate were the maximum in CIM- 616 and phosphorus treated with $100 \mathrm{~kg} \mathrm{ha}^{-1}\left(\mathrm{~T}_{2}\right)$, while leaf area index as well as crop growth rate was the minimum in cultivar FH-LALAZAR treated as control $\left(\mathrm{T}_{\mathrm{o}}\right)$. Net assimilation rate was the maximum in cultivar CIM-612 (1.480) and the phosphorus level was treated as $150 \mathrm{kgha}^{-1}$ $\left(\mathrm{T}_{3}\right)$. Leaf area duration and phosphorus concentration were the highest in cultivar MNH-886 (871.33) which treated with phosphorus level of $150 \mathrm{kgha}^{-1}\left(\mathrm{~T}_{3}\right)$ of phosphorus. The maximum fiber uniformity, staple length, fiber strength were in cultivar MNH-886 (70.667) and treatment was $150 \mathrm{~kg} \mathrm{ha}^{-1}\left(\mathrm{~T}_{3}\right)$. Micronair value was the maximum in cultivar CIM616 (5.166) and phosphorus level was 150 $\mathrm{kg} \mathrm{ha}^{-1}$. Current study in line to previous work because supplemental application of phosphorus improves yield, quality and net returns of cotton [22-24]. 

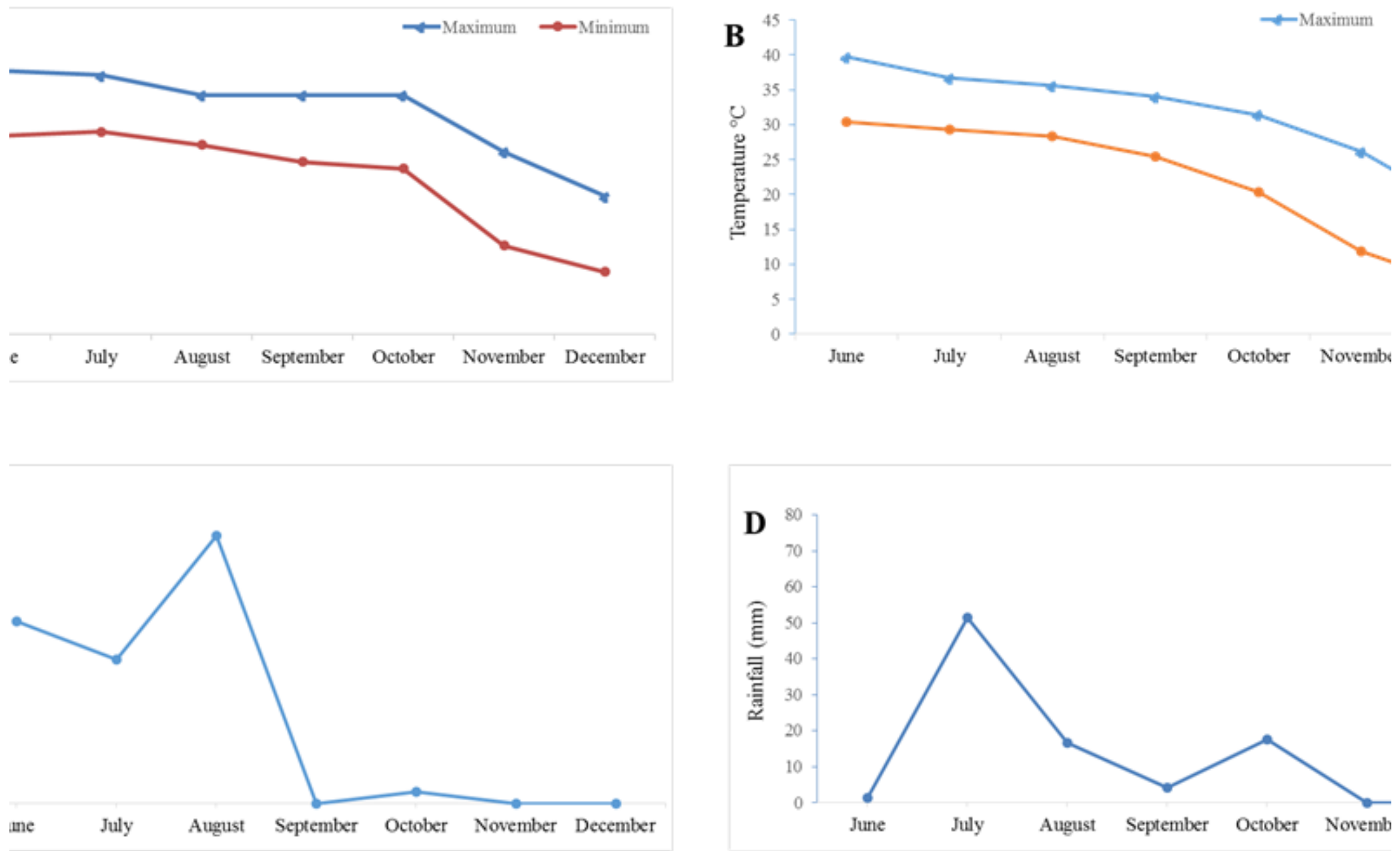

Figure 1. Eco-meteorological data of Multan, Punjab (Pakistan) during 2015 (A \& C) and during 2016 (B \& D) 
Table 1. Significance level of agronomic, yield and fiber related traits as affected by cultivars and phosphorus concentrations

\begin{tabular}{|c|c|c|c|}
\hline Parameters & $\begin{array}{c}\text { Cotton } \\
\text { cultivars }\end{array}$ & $\begin{array}{l}\text { Phosphorus } \\
\text { application }\end{array}$ & $\begin{array}{c}\text { Cotton cultivars } \times \\
\text { phosphorus application }\end{array}$ \\
\hline Plant height $(\mathrm{cm})$ & $352.500 * *$ & $464.222 * *$ & $7.167 * *$ \\
\hline Number of bolls plant $t^{-1}$ & $53.8917 * *$ & $77.2667 * *$ & $1.2806^{* *}$ \\
\hline Number of nodes plant ${ }^{-1}$ & $44.2667 * *$ & $46.8167 * *$ & $2.4000 \mathrm{~ns}$ \\
\hline Boll weight (g) & $0.39208 * *$ & $1.12044 * *$ & $0.19586 * *$ \\
\hline Lint yield $\left(\mathrm{kg} \mathrm{ha}^{-1}\right)$ & $552.442 * *$ & $118.333^{* *}$ & $16.264 * *$ \\
\hline Biological yield $\left(\mathrm{kg} \mathrm{ha}^{-1}\right)$ & $0.06262 * *$ & $0.18012 * *$ & $0.00310 * *$ \\
\hline Seed cotton harvest $(\%)$ & $156.659 * *$ & $200.623 \mathrm{~ns}$ & $5.546^{* *}$ \\
\hline Ginning out turn (\%) & $2.244 * *$ & $1.350 \mathrm{~ns}$ & $3.711 \mathrm{~ns}$ \\
\hline Leaf area index & $0.20759 * *$ & $0.02123^{* *}$ & $0.01455^{* *}$ \\
\hline Crop growth rate $\left(\mathrm{g} \mathrm{m}^{-2}\right.$ day $\left.^{-1}\right)$ & $0.31000 * *$ & $0.17217 * *$ & $0.24411 * *$ \\
\hline Net assimilation rate $\left(\mathrm{g} \mathrm{m}^{-2}\right.$ week $\left.^{-1}\right)$ & $0.01003 * *$ & $0.01784 * *$ & $0.00232 * *$ \\
\hline Leaf area duration (days) & $0.01003 * *$ & $0.01784 * *$ & $0.00232 * *$ \\
\hline Phosphorus concentration & $0.20759 * *$ & $0.02123^{* *}$ & $0.01455^{* *}$ \\
\hline Fiber uniformity & $589.142 * *$ & $466.000 * *$ & $71.986^{* *}$ \\
\hline Staple length (mm) & $651.608^{* *}$ & $125.022 * *$ & $34.953^{* *}$ \\
\hline Fiber strength (tppsi) & $7.889 \mathrm{~ns}$ & $105.933^{* *}$ & $34.194 * *$ \\
\hline $\operatorname{Micronair}\left(\mu\right.$ inch $\left.^{-1}\right)$ & $0.29142 * *$ & $0.16950 * *$ & $0.24411 * *$ \\
\hline
\end{tabular}

$\mathrm{NS}=$ non-significant; $*=$ significant $(\mathrm{P}<0.05)$ and $* *=$ highly significant $(\mathrm{P}<0.01)$ 
Table 2. Individual effect of cultivars on agronomic and yield related traits

\begin{tabular}{|c|c|c|c|c|c|c|c|c|c|}
\hline Cultivars & $\begin{array}{l}\text { Plant height } \\
(\mathrm{cm})\end{array}$ & $\begin{array}{l}\text { Number of } \\
\text { bolls/plant }\end{array}$ & $\begin{array}{c}\text { Number of } \\
\text { nodes/plant }\end{array}$ & $\begin{array}{c}\text { Boll weight } \\
(\mathrm{g})\end{array}$ & $\begin{array}{c}\text { Seed cotton } \\
\text { yield }\left(\mathrm{kg} \mathrm{ha}^{-1}\right)\end{array}$ & $\begin{array}{c}\text { Lint yield ( kg } \\
\left.\text { ha }^{-1}\right)\end{array}$ & $\begin{array}{c}\text { Biological yield } \\
\left(\mathrm{kg} \mathrm{ha}^{-1}\right)\end{array}$ & $\begin{array}{l}\text { Seed cotton } \\
\text { harvest }(\%)\end{array}$ & $\begin{array}{c}\text { Ginning out } \\
\text { turn (\%) }\end{array}$ \\
\hline CIM-598 & $146.67 \mathrm{~b}$ & $23.00 \mathrm{c}$ & $40.58 \mathrm{~b}$ & $1.49 \mathrm{~b}$ & $2289.0 \mathrm{a}$ & $41.41 \mathrm{c}$ & $0.83 \mathrm{ab}$ & $22 \mathrm{a}$ & $0.020 \mathrm{ab}$ \\
\hline MNH-886 & $152.50 \mathrm{a}$ & $25.66 \mathrm{~b}$ & $40.58 \mathrm{~b}$ & $1.45 \mathrm{~b}$ & $2138.8 \mathrm{a}$ & $53.50 \mathrm{a}$ & $0.58 \mathrm{c}$ & $21 \mathrm{a}$ & $0.017 \mathrm{c}$ \\
\hline CIM-616 & $152.50 \mathrm{a}$ & $28.03 \mathrm{a}$ & $44.91 \mathrm{a}$ & $1.20 \mathrm{c}$ & $2161.9 \mathrm{a}$ & $39.33 \mathrm{~d}$ & $0.81 \mathrm{ab}$ & $21 \mathrm{a}$ & $0.020 \mathrm{ab}$ \\
\hline CIM-612 & $141.67 \mathrm{c}$ & $24.75 \mathrm{~b}$ & $40.75 \mathrm{~b}$ & $1.48 \mathrm{~b}$ & $2354.1 \mathrm{a}$ & $47.33 \mathrm{~b}$ & $0.89 \mathrm{a}$ & $23 \mathrm{a}$ & $0.019 \mathrm{~b}$ \\
\hline FH-lalazar & $141.67 \mathrm{c}$ & $23.00 \mathrm{c}$ & $40.58 \mathrm{~b}$ & $1.70 \mathrm{a}$ & $2590.5 \mathrm{a}$ & $36.58 \mathrm{e}$ & $0.79 \mathrm{~b}$ & $25 \mathrm{a}$ & $0.021 \mathrm{a}$ \\
\hline
\end{tabular}

Mean values sharing similar letter(s) in a column are statistically non-significant at $p=0.05$ (LSD test)

Table 3. Individual effect of cultivars on physiological and fiber quality traits

\begin{tabular}{|c|c|c|c|c|c|c|c|c|c|}
\hline Cultivars & $\begin{array}{c}\text { Leaf } \\
\text { area } \\
\text { index }\end{array}$ & $\begin{array}{c}\text { Crop growth } \\
\text { rate }\left(\mathrm{g} \mathrm{m}^{-2} \text { day }^{-1}\right)\end{array}$ & $\begin{array}{l}\text { Net assimilation } \\
\text { rate }\left(\mathrm{g} \mathrm{m}^{-2} \text { week }^{-1}\right)\end{array}$ & $\begin{array}{c}\text { Leaf area } \\
\text { duration (days) }\end{array}$ & $\begin{array}{l}\text { Phosphorus } \\
\text { concentration }\end{array}$ & $\begin{array}{c}\text { Fiber } \\
\text { uniformity }\end{array}$ & $\begin{array}{l}\text { Staple length } \\
(\mathrm{mm})\end{array}$ & $\begin{array}{l}\text { Fiber strength } \\
\quad \text { (tppsi) }\end{array}$ & $\begin{array}{l}\text { Micronair }(\mu \\
\left.\text { inch }^{-1}\right)\end{array}$ \\
\hline CIM-598 & $0.58 \mathrm{c}$ & $4.28 \mathrm{bc}$ & $1.31 \mathrm{c}$ & $546.5 \mathrm{c}$ & $40.25 \mathrm{c}$ & $40.25 \mathrm{c}$ & $21.75 \mathrm{c}$ & $24.83 \mathrm{c}$ & $4.28 \mathrm{bc}$ \\
\hline MNH-886 & $0.70 \mathrm{~b}$ & $4.30 \mathrm{bc}$ & $1.38 \mathrm{a}$ & $760.1 \mathrm{a}$ & $48.16 \mathrm{a}$ & $58.16 \mathrm{a}$ & $36.16 \mathrm{a}$ & $30.66 \mathrm{a}$ & $4.30 \mathrm{bc}$ \\
\hline CIM-616 & $0.80 \mathrm{a}$ & $4.63 \mathrm{a}$ & $1.37 \mathrm{ab}$ & $461.7 \mathrm{~d}$ & $45.83 \mathrm{~b}$ & $45.83 \mathrm{~b}$ & $19.58 \mathrm{~d}$ & $24.50 \mathrm{c}$ & $4.63 \mathrm{a}$ \\
\hline CIM-612 & $0.81 \mathrm{a}$ & $4.25 \mathrm{~b}$ & $1.36 \mathrm{~b}$ & $637.5 \mathrm{~b}$ & $45.75 \mathrm{~b}$ & $45.75 \mathrm{~b}$ & $24.50 \mathrm{~b}$ & $28.16 \mathrm{~b}$ & $4.25 \mathrm{~b}$ \\
\hline FH-lalazar & $0.51 \mathrm{~d}$ & $4.23 \mathrm{c}$ & $1.32 \mathrm{c}$ & $404.2 \mathrm{e}$ & $42.00 \mathrm{c}$ & $42.00 \mathrm{c}$ & $17.33 \mathrm{e}$ & $23.58 \mathrm{c}$ & $4.23 \mathrm{c}$ \\
\hline
\end{tabular}

Mean values sharing similar letter(s) in a column are statistically non-significant at $p=0.05$ (LSD test) 
Table 4. Individual effect of phosphorus on agronomic and yield related traits

\begin{tabular}{|c|c|c|c|c|c|c|c|c|c|}
\hline Treatment & $\begin{array}{c}\text { Plant height } \\
(\mathrm{cm})\end{array}$ & $\begin{array}{l}\text { Number of } \\
\text { bolls/ plant }\end{array}$ & $\begin{array}{c}\text { Number of } \\
\text { nodes/ plant }\end{array}$ & $\begin{array}{c}\text { Boll weight } \\
(\mathrm{g})\end{array}$ & $\begin{array}{c}\text { Seed cotton } \\
\text { yield }\left(\mathrm{kg} \mathrm{ha}^{-1}\right)\end{array}$ & $\begin{array}{c}\text { Lint yield ( } \\
\mathrm{kg} \mathrm{ha}^{-1} \text { ) }\end{array}$ & $\begin{array}{c}\text { Biological } \\
\text { yield }\left(\mathrm{kg} \mathrm{ha}^{-1}\right)\end{array}$ & $\begin{array}{l}\text { Seed cotton } \\
\text { harvest }(\%)\end{array}$ & $\begin{array}{c}\text { Ginning out } \\
\text { turn }(\%)\end{array}$ \\
\hline Control $\left(\mathrm{P}_{0}\right)$ & $140.73 \mathrm{~d}$ & $22.33 \mathrm{~d}$ & $39.26 \mathrm{c}$ & $1.30 \mathrm{~b}$ & $2116.9 \mathrm{a}$ & $40.40 \mathrm{c}$ & $0.73 \mathrm{~b}$ & $21 \mathrm{a}$ & $0.018 \mathrm{a}$ \\
\hline $50 \mathrm{~kg} / \mathrm{ha}\left(\mathrm{P}_{1}\right)$ & $145.13 \mathrm{c}$ & $24.13 \mathrm{c}$ & $40.93 \mathrm{~b}$ & $1.30 \mathrm{~b}$ & $2221.3 \mathrm{a}$ & $43.00 \mathrm{~b}$ & $0.75 \mathrm{~b}$ & $22 \mathrm{a}$ & $0.018 \mathrm{a}$ \\
\hline $100 \mathrm{~kg} / \mathrm{ha}\left(\mathrm{P}_{2}\right)$ & $149.13 \mathrm{~b}$ & $25.40 \mathrm{~b}$ & $42.46 \mathrm{a}$ & $1.38 \mathrm{~b}$ & $2221.4 \mathrm{a}$ & $43.93 \mathrm{~b}$ & $0.80 \mathrm{ab}$ & $22 \mathrm{a}$ & $0.018 \mathrm{a}$ \\
\hline $150 \mathrm{~kg} / \mathrm{ha}\left(\mathrm{P}_{3}\right)$ & $153.87 \mathrm{a}$ & $27.73 \mathrm{a}$ & $43.26 \mathrm{a}$ & $1.47 \mathrm{a}$ & $2667.9 \mathrm{a}$ & $47.29 \mathrm{a}$ & $0.83 \mathrm{a}$ & $26 \mathrm{a}$ & $0.019 \mathrm{a}$ \\
\hline
\end{tabular}

Mean values sharing similar letter(s) in a column are statistically non-significant at $p=0.05$ (LSD test)

Table 5. Individual effect of phosphorus on physiological and fiber related traits

\begin{tabular}{|c|c|c|c|c|c|c|c|c|c|}
\hline Treatment & $\begin{array}{l}\text { Leaf } \\
\text { area } \\
\text { index }\end{array}$ & $\begin{array}{c}\text { Crop growth } \\
\text { rate }\left(\mathrm{g} \mathrm{m}^{-2} \text { day }^{-1}\right)\end{array}$ & $\begin{array}{l}\text { Net assimilation } \\
\text { rate }\left(\mathrm{g} \mathrm{m}^{-2} \text { week }^{-1}\right)\end{array}$ & $\begin{array}{c}\text { Leaf area } \\
\text { duration (days) }\end{array}$ & $\begin{array}{l}\text { Phosphorus } \\
\text { concentration }\end{array}$ & $\begin{array}{c}\text { Fiber } \\
\text { uniformity }\end{array}$ & $\begin{array}{c}\text { Staple } \\
\text { length } \\
(\mathrm{mm})\end{array}$ & $\begin{array}{c}\text { Fiber } \\
\text { strength } \\
\text { (tppsi) }\end{array}$ & $\begin{array}{l}\text { Micronair } \\
\left(\mu \text { inch }^{-1}\right)\end{array}$ \\
\hline Control $\left(\mathrm{P}_{0}\right)$ & $0.64 \mathrm{c}$ & $4.24 \mathrm{~b}$ & $1.31 \mathrm{~d}$ & $495.9 \mathrm{~d}$ & $41.00 \mathrm{c}$ & $41.00 \mathrm{c}$ & $20.33 d$ & $24.73 \mathrm{~b}$ & $4.24 \mathrm{~b}$ \\
\hline $50 \mathrm{~kg} \mathrm{ha}^{-1}\left(\mathrm{P}_{1}\right)$ & $0.66 \mathrm{~b}$ & $4.32 \mathrm{ab}$ & $1.33 \mathrm{c}$ & $538.1 \mathrm{c}$ & $44.80 \mathrm{~b}$ & $44.80 \mathrm{~b}$ & $23.40 \mathrm{c}$ & $25.46 \mathrm{~b}$ & $4.32 \mathrm{ab}$ \\
\hline $100 \mathrm{~kg} \mathrm{ha}^{-1}\left(\mathrm{P}_{2}\right)$ & $0.71 \mathrm{a}$ & $4.46 \mathrm{a}$ & $1.35 \mathrm{~b}$ & $582.6 \mathrm{~b}$ & $45.60 \mathrm{~b}$ & $45.60 \mathrm{~b}$ & $24.40 \mathrm{~b}$ & $27.20 \mathrm{a}$ & $4.46 \mathrm{a}$ \\
\hline $150 \mathrm{~kg} \mathrm{ha}^{-1}\left(\mathrm{P}_{3}\right)$ & $0.71 \mathrm{a}$ & $4.46 \mathrm{a}$ & $1.39 \mathrm{a}$ & $631.4 \mathrm{a}$ & $54.20 \mathrm{a}$ & $54.20 \mathrm{a}$ & $27.33 \mathrm{a}$ & $28.00 \mathrm{a}$ & $4.46 \mathrm{a}$ \\
\hline
\end{tabular}

Mean values sharing similar letter(s) in a column are statistically non-significant at $p=0.05$ (LSD test) 
Table 6. Interactive effect of phosphorus and cotton cultivars on agronomic and yield related traits

\begin{tabular}{|c|c|c|c|c|c|c|c|c|c|}
\hline Treatment & $\begin{array}{c}\text { Plant height } \\
(\mathbf{c m})\end{array}$ & $\begin{array}{l}\text { Number of } \\
\text { bolls/ plant }\end{array}$ & $\begin{array}{c}\text { Number of } \\
\text { nodes/ plant }\end{array}$ & $\begin{array}{c}\text { Boll weight } \\
(\mathrm{g})\end{array}$ & $\begin{array}{c}\text { Seed cotton } \\
\text { yield }\left(\mathrm{kg} \mathrm{ha}^{-1}\right)\end{array}$ & $\begin{array}{c}\text { Lint yield (kg } \\
\left.\text { ha }^{-1}\right)\end{array}$ & $\begin{array}{c}\text { Biological yield } \\
\left(\mathrm{kg} \mathrm{ha}^{-1}\right)\end{array}$ & $\begin{array}{l}\text { Seed cotton } \\
\text { harvest }(\%)\end{array}$ & $\begin{array}{c}\text { Ginning out } \\
\text { turn }(\%)\end{array}$ \\
\hline $\mathbf{P}_{0} \mathbf{V}_{1}$ & $140.67 \mathrm{~h}$ & $20.500 \mathrm{~h}$ & $38.667 \mathrm{~h}$ & $1.133 \mathrm{gh}$ & $2091.3 \mathrm{de}$ & $39.00 \mathrm{~g}$ & $0.800 \mathrm{c}-\mathrm{f}$ & 880.0 a-c & $0.021 \mathrm{bc}$ \\
\hline $\mathbf{P}_{1} \mathbf{V}_{1}$ & $144.67 \mathrm{f}$ & $22.000 \mathrm{~g}$ & $40.500 \mathrm{fg}$ & $1.333 \mathrm{de}$ & $2174.7 \mathrm{~d}$ & $41.333 \mathrm{f}$ & $0.832 \mathrm{c}-\mathrm{e}$ & $2174.7 \mathrm{~b}-\mathrm{d}$ & $0.020 \mathrm{bc}$ \\
\hline$P_{2} V_{1}$ & $148.83 \mathrm{~d}$ & $23.833 \mathrm{f}$ & $41.667 \mathrm{de}$ & $1.400 \mathrm{~cd}$ & $2428.3 \mathrm{bc}$ & $41.333 \mathrm{f}$ & $0.830 \mathrm{c}-\mathrm{e}$ & $2428.3 \mathrm{a}-\mathrm{d}$ & $0.020 \mathrm{bc}$ \\
\hline $\mathbf{P}_{3} \mathbf{V}_{1}$ & $152.83 \mathrm{c}$ & $26.000 \mathrm{~d}$ & $41.833 \mathrm{~d}$ & $2.133 \mathrm{a}$ & $2471.7 \mathrm{bc}$ & $44.500 \mathrm{e}$ & $0.865 \mathrm{~b}-\mathrm{d}$ & $2471.7 \mathrm{a}-\mathrm{d}$ & $0.019 b c$ \\
\hline $\mathbf{P}_{0} \mathrm{~V}_{2}$ & $144.83 \mathrm{f}$ & $24.000 \mathrm{f}$ & $38.667 \mathrm{~h}$ & $1.150 \mathrm{f}-\mathrm{h}$ & $1923.7 \mathrm{fg}$ & $48.333 \mathrm{~d}$ & $0.527 \mathrm{~h}$ & $2928.3 \mathrm{ab}$ & $0.011 \mathrm{e}$ \\
\hline $\mathbf{P}_{1} \mathbf{V}_{2}$ & $149.33 \mathrm{~d}$ & $25.833 \mathrm{de}$ & $40.667 \mathrm{e}-\mathrm{g}$ & $1.333 \mathrm{de}$ & $1831.3 \mathrm{gh}$ & $54.167 \mathrm{c}$ & $0.492 \mathrm{~h}$ & $1831.3 \mathrm{~cd}$ & $0.009 \mathrm{f}$ \\
\hline $\mathbf{P}_{2} \mathbf{V}_{2}$ & $155.17 \mathrm{~b}$ & $26.000 \mathrm{~d}$ & $41.667 \mathrm{de}$ & $1.333 \mathrm{de}$ & $1903.7 \mathrm{fg}$ & $55.333 \mathrm{bc}$ & $0.665 \mathrm{~g}$ & 1903.7 b-d & $0.012 \mathrm{e}$ \\
\hline $\mathbf{P}_{3} \mathbf{V}_{2}$ & $161.33 \mathrm{a}$ & $27.167 \mathrm{c}$ & $41.833 \mathrm{~d}$ & $2.083 \mathrm{a}$ & $2928.3 \mathrm{a}$ & $56.667 \mathrm{a}$ & $0.6667 \mathrm{~g}$ & 1923.7 b-d & $0.012 \mathrm{e}$ \\
\hline $\mathbf{P}_{0} \mathrm{~V}_{3}$ & $144.83 \mathrm{f}$ & $25.000 \mathrm{e}$ & $41.667 \mathrm{de}$ & $1.033 \mathrm{~h}$ & 2027.7 ef & $37.667 \mathrm{hi}$ & $0.790 \mathrm{~d}-\mathrm{f}$ & 2027.7 b-d & $0.020 \mathrm{a}-\mathrm{c}$ \\
\hline $\mathbf{P}_{1} \mathbf{V}_{3}$ & $149.33 \mathrm{~d}$ & $27.667 \mathrm{bc}$ & $43.500 \mathrm{c}$ & $1.200 \mathrm{e}-\mathrm{h}$ & $2350.7 \mathrm{c}$ & $39.500 \mathrm{~g}$ & $0.840 \mathrm{c}-\mathrm{e}$ & $2350.7 \mathrm{~b}-\mathrm{d}$ & $0.021 \mathrm{ab}$ \\
\hline $\mathbf{P}_{2} \mathbf{V}_{3}$ & $155.17 \mathrm{~b}$ & $28.500 \mathrm{~b}$ & $45.833 \mathrm{~b}$ & $1.366 \mathrm{de}$ & $2128.0 \mathrm{de}$ & $39.333 \mathrm{~g}$ & $0.801 \mathrm{c}-\mathrm{f}$ & $2128.0 \mathrm{~b}-\mathrm{d}$ & $0.020 \mathrm{bc}$ \\
\hline $\mathbf{P}_{3} \mathbf{V}_{3}$ & $161.33 \mathrm{a}$ & $31.667 \mathrm{a}$ & $49.000 \mathrm{a}$ & $1.283 \mathrm{~d}-\mathrm{g}$ & $2174.7 \mathrm{~d}$ & $41.667 \mathrm{f}$ & $0.830 \mathrm{c}-\mathrm{e}$ & $2174.7 \mathrm{~b}-\mathrm{d}$ & $0.019 \mathrm{bc}$ \\
\hline $\mathbf{P}_{0} \mathbf{V}_{4}$ & $136.17 \mathrm{i}$ & $22.000 \mathrm{~g}$ & $38.500 \mathrm{~h}$ & $1.200 \mathrm{e}-\mathrm{h}$ & $1648.01 \mathrm{j}$ & $41.000 \mathrm{f}$ & $0.828 \mathrm{c}-\mathrm{e}$ & $2361.7 \mathrm{~b}-\mathrm{d}$ & $0.020 \mathrm{bc}$ \\
\hline $\mathbf{P}_{1} \mathbf{V}_{4}$ & $141.00 \mathrm{~h}$ & $23.500 \mathrm{f}$ & $40.333 \mathrm{~g}$ & $1.316 \mathrm{~d}-\mathrm{f}$ & $2495.0 \mathrm{~b}$ & $44.167 \mathrm{e}$ & $0.866 \mathrm{~b}-\mathrm{d}$ & $2495.0 \mathrm{a}-\mathrm{d}$ & $0.019 \mathrm{bc}$ \\
\hline $\mathbf{P}_{2} \mathbf{V}_{4}$ & $143.00 \mathrm{~g}$ & $25.333 \mathrm{de}$ & $41.500 \mathrm{~d}-\mathrm{f}$ & $1.550 \mathrm{c}$ & $2361.7 \mathrm{c}$ & $48.333 \mathrm{~d}$ & $0.926 \mathrm{ab}$ & $2878.3 \mathrm{a}-\mathrm{c}$ & $0.019 \mathrm{~cd}$ \\
\hline $\mathbf{P}_{3} \mathbf{V}_{4}$ & $146.33 \mathrm{e}$ & $28.000 \mathrm{bc}$ & $42.333 \mathrm{~d}$ & $1.833 \mathrm{~b}$ & $2878.3 \mathrm{a}$ & $55.667 \mathrm{ab}$ & $0.960 \mathrm{a}$ & $1648.0 \mathrm{~d}$ & $0.017 \mathrm{~d}$ \\
\hline $\mathbf{P}_{0} \mathrm{~V}_{5}$ & $136.50 \mathrm{i}$ & $20.333 \mathrm{~h}$ & $38.667 \mathrm{~h}$ & $2.050 \mathrm{a}$ & $1698.0 \mathrm{ij}$ & $36.167 \mathrm{j}$ & $0.730 \mathrm{fg}$ & $1698.0 \mathrm{~d}$ & $0.020 \mathrm{bc}$ \\
\hline $\mathbf{P}_{1} \mathbf{V}_{5}$ & $141.17 \mathrm{~h}$ & $22.167 \mathrm{~g}$ & $40.667 \mathrm{e}-\mathrm{g}$ & $1.333 \mathrm{de}$ & $1763.0 \mathrm{~h}-\mathrm{j}$ & $36.167 \mathrm{j}$ & $0.766 \mathrm{ef}$ & $1763.0 \mathrm{~d}$ & $0.021 \mathrm{ab}$ \\
\hline $\mathbf{P}_{2} \mathbf{V}_{5}$ & $143.00 \mathrm{~g}$ & $23.667 \mathrm{f}$ & $41.667 \mathrm{de}$ & $1.333 \mathrm{de}$ & $1801.3 \mathrm{~g}-\mathrm{i}$ & $36.667 \mathrm{ij}$ & 0.8233 c-e & $1801.3 \mathrm{~d}$ & $0.0227 \mathrm{a}$ \\
\hline $\mathbf{P}_{3} \mathbf{V}_{5}$ & $146.67 \mathrm{e}$ & $26.167 \mathrm{~d}$ & $42.167 \mathrm{~d}$ & $2.200 \mathrm{a}$ & $1814.7 \mathrm{~g}-\mathrm{i}$ & $38.500 \mathrm{gh}$ & $0.880 \mathrm{a}-\mathrm{c}$ & $3481.3 \mathrm{a}$ & $0.022 \mathrm{a}$ \\
\hline
\end{tabular}

Mean values sharing similar letter(s) in a column are statistically non-significant at $\mathrm{p}=0.05$ (LSD test) 
Ahmad et al.

Table 7. Effect of phosphorus on physiological and fiber quality traits of cotton cultivars

\begin{tabular}{|c|c|c|c|c|c|c|c|c|c|}
\hline Treatment & $\begin{array}{l}\text { Leaf area } \\
\text { index }\end{array}$ & $\begin{array}{l}\text { Crop growth rate } \\
\left(\mathrm{g} \mathrm{m}^{-2} \text { day }^{-1}\right)\end{array}$ & $\begin{array}{l}\text { Net assimilation } \\
\text { rate }\left(\mathrm{g} \mathrm{m}^{-2} \text { week }^{-1}\right)\end{array}$ & $\begin{array}{c}\text { Leaf area } \\
\text { duration (days) }\end{array}$ & $\begin{array}{l}\text { Phosphorus } \\
\text { concentration }\end{array}$ & $\begin{array}{c}\text { Fiber } \\
\text { uniformity }\end{array}$ & $\begin{array}{c}\text { Staple length } \\
(\mathrm{mm})\end{array}$ & $\begin{array}{c}\text { Fiber strength } \\
\text { (tppsi) }\end{array}$ & $\begin{array}{c}\text { Micronair ( } \mu \\
\left.\text { inch }^{-1}\right)\end{array}$ \\
\hline $\mathbf{P}_{0} \mathrm{~V}_{1}$ & $0.558 \mathrm{k}$ & 0.725 o & $0.768 \mathrm{mn}$ & $492.67 \mathrm{~g}$ & $0.185 \mathrm{gh}$ & $39.667 \mathrm{gh}$ & $20.000 \mathrm{~g}$ & $24.333 \mathrm{de}$ & $4.066 \mathrm{f}$ \\
\hline $\mathbf{P}_{1} \mathbf{V}_{1}$ & $0.5700 \mathrm{j}$ & $0.751 \mathrm{n}$ & $1.315 \mathrm{~h}$ & $515.67 \mathrm{f}$ & $0.196 \mathrm{e}-\mathrm{h}$ & $39.333 \mathrm{~h}$ & $20.333 \mathrm{fg}$ & $25.000 \mathrm{de}$ & $4.166 \mathrm{ef}$ \\
\hline $\mathbf{P}_{2} \mathbf{V}_{1}$ & $0.590 \mathrm{i}$ & $0.785 \mathrm{~m}$ & $1.331 \mathrm{fg}$ & $584.67 \mathrm{e}$ & $0.201 \mathrm{~d}-\mathrm{h}$ & $40.500 \mathrm{f}-\mathrm{h}$ & $22.833 \mathrm{de}$ & $25.833 \mathrm{~cd}$ & $4.416 \mathrm{~cd}$ \\
\hline $\mathbf{P}_{3} \mathbf{V}_{1}$ & $0.618 \mathrm{~h}$ & 0.8251 & $1.336 \mathrm{f}$ & $594.67 \mathrm{de}$ & $0.205 \mathrm{c}-\mathrm{h}$ & $41.667 \mathrm{e}-\mathrm{g}$ & $24.000 \mathrm{~d}$ & $24.333 \mathrm{de}$ & $4.500 \mathrm{c}$ \\
\hline $\mathbf{P}_{0} \mathbf{V}_{2}$ & $0.680 \mathrm{~g}$ & $0.903 \mathrm{k}$ & $1.330 \mathrm{fg}$ & $613.33 \mathrm{~d}$ & $0.220 \mathrm{~b}-\mathrm{g}$ & $43.500 \mathrm{e}$ & $25.833 \mathrm{c}$ & $27.167 \mathrm{c}$ & $4.750 \mathrm{~b}$ \\
\hline$P_{1} V_{2}$ & $0.681 \mathrm{~g}$ & $0.940 \mathrm{j}$ & $1.380 \mathrm{~d}$ & $755.67 \mathrm{c}$ & $0.2250 \mathrm{~b}-\mathrm{f}$ & $57.333 \mathrm{c}$ & $35.333 \mathrm{~b}$ & $29.000 \mathrm{~b}$ & $4.066 \mathrm{f}$ \\
\hline $\mathbf{P}_{2} \mathbf{V}_{2}$ & $0.711 \mathrm{f}$ & $0.993 \mathrm{hi}$ & $1.398 \mathrm{c}$ & $805.33 b$ & $0.231 \mathrm{a}-\mathrm{e}$ & $62.000 \mathrm{~b}$ & $36.500 \mathrm{~b}$ & $33.500 \mathrm{a}$ & $4.200 \mathrm{ef}$ \\
\hline $\mathbf{P}_{3} \mathbf{V}_{2}$ & $0.743 \mathrm{e}$ & $1.056 \mathrm{c}$ & $1.421 \mathrm{~b}$ & $871.33 \mathrm{a}$ & $0.241 \mathrm{a}-\mathrm{c}$ & $70.667 \mathrm{a}$ & $47.500 \mathrm{a}$ & $33.500 \mathrm{a}$ & $4.250 \mathrm{de}$ \\
\hline $\mathbf{P}_{0} \mathrm{~V}_{3}$ & $0.768 \mathrm{~cd}$ & $1.026 \mathrm{ef}$ & $1.336 \mathrm{f}$ & $415.33 \mathrm{hi}$ & $0.248 \mathrm{ab}$ & 42.33 ef & $17.667 \mathrm{~h}$ & $23.333 \mathrm{e}$ & $4.266 \mathrm{de}$ \\
\hline $\mathbf{P}_{1} \mathbf{V}_{3}$ & $0.776 \mathrm{c}$ & $1.050 \mathrm{~cd}$ & $1.350 \mathrm{e}$ & $430.00 \mathrm{~h}$ & $0.270 \mathrm{a}$ & $45.667 \mathrm{~d}$ & $20.833 \mathrm{fg}$ & $24.667 \mathrm{de}$ & $4.416 \mathrm{~cd}$ \\
\hline $\mathbf{P}_{2} \mathbf{V}_{3}$ & $0.980 \mathrm{a}$ & $1.361 \mathrm{~b}$ & $1.388 \mathrm{~cd}$ & $494.33 \mathrm{fg}$ & $0.178 \mathrm{~h}$ & $40.000 \mathrm{gh}$ & $20.333 \mathrm{fg}$ & $25.000 \mathrm{de}$ & $4.733 \mathrm{~b}$ \\
\hline $\mathbf{P}_{\mathbf{3}} \mathbf{V}_{3}$ & $0.708 \mathrm{f}$ & $1.003 \mathrm{gh}$ & $1.413 \mathrm{~b}$ & $512.33 \mathrm{fg}$ & $0.186 \mathrm{f}-\mathrm{h}$ & $56.000 \mathrm{c}$ & $21.333 \mathrm{f}$ & $25.667 \mathrm{~cd}$ & $5.166 \mathrm{a}$ \\
\hline $\mathbf{P}_{0} \mathrm{~V}_{4}$ & $0.740 \mathrm{e}$ & $0.983 \mathrm{i}$ & $1.330 \mathrm{fg}$ & $588.00 \mathrm{e}$ & $0.190 \mathrm{f}-\mathrm{h}$ & $40.333 \mathrm{f}-\mathrm{h}$ & $22.667 \mathrm{e}$ & $25.667 \mathrm{~cd}$ & $4.400 \mathrm{~cd}$ \\
\hline $\mathbf{P}_{1} \mathbf{V}_{4}$ & $0.765 \mathrm{~d}$ & $1.016 \mathrm{fg}$ & $1.331 \mathrm{fg}$ & $593.00 \mathrm{de}$ & $0.201 \mathrm{~d}-\mathrm{h}$ & $41.667 \mathrm{e}-\mathrm{g}$ & $24.000 \mathrm{~d}$ & $25.667 \mathrm{~cd}$ & $4.500 \mathrm{c}$ \\
\hline $\mathbf{P}_{2} \mathbf{V}_{4}$ & $0.776 \mathrm{c}$ & $1.038 \mathrm{de}$ & $1.333 \mathrm{f}$ & $611.67 \mathrm{~d}$ & $0.218 \mathrm{~b}-\mathrm{g}$ & $43.333 \mathrm{e}$ & $25.667 \mathrm{c}$ & $29.000 \mathrm{~b}$ & $4.733 \mathrm{~b}$ \\
\hline $\mathbf{P}_{3} \mathbf{V}_{4}$ & $0.956 \mathrm{~b}$ & $1.416 \mathrm{a}$ & $1.480 \mathrm{a}$ & $755.67 \mathrm{c}$ & $0.236 \mathrm{a}-\mathrm{d}$ & $57.667 \mathrm{c}$ & $25.667 \mathrm{c}$ & $32.333 \mathrm{a}$ & $4.066 \mathrm{f}$ \\
\hline$P_{0} V_{5}$ & $0.4733 \mathrm{n}$ & $0.615 \mathrm{q}$ & $1.298 \mathrm{i}$ & $372.00 \mathrm{j}$ & $0.230 \mathrm{~b}-\mathrm{e}$ & $39.667 \mathrm{gh}$ & $16.000 \mathrm{i}$ & $23.667 \mathrm{e}$ & $4.200 \mathrm{ef}$ \\
\hline$P_{1} V_{5}$ & $0.5117 \mathrm{~m}$ & $0.676 \mathrm{p}$ & $1.321 \mathrm{gh}$ & $406.33 \mathrm{i}$ & $0.240 \mathrm{a}-\mathrm{d}$ & $40.500 \mathrm{f}-\mathrm{h}$ & $17.167 \mathrm{hi}$ & $23.333 \mathrm{e}$ & 4.150 ef \\
\hline $\mathbf{P}_{2} \mathbf{V}_{5}$ & 0.5311 & $0.710 \mathrm{o}$ & $1.336 \mathrm{f}$ & $417.00 \mathrm{hi}$ & $0.250 \mathrm{ab}$ & $43.167 \mathrm{e}$ & $17.500 \mathrm{~h}$ & $23.500 \mathrm{e}$ & $4.283 \mathrm{de}$ \\
\hline $\mathbf{P}_{3} \mathbf{V}_{5}$ & $0.565 \mathrm{jk}$ & $0.768 \mathrm{mn}$ & $1.355 \mathrm{e}$ & $431.67 \mathrm{~h}$ & $0.270 \mathrm{a}$ & $45.667 \mathrm{~d}$ & $19.833 \mathrm{~g}$ & $24.667 \mathrm{de}$ & $4.400 \mathrm{~cd}$ \\
\hline
\end{tabular}

Mean values sharing similar letter(s) in a column are statistically non-significant at $\mathrm{p}=0.05$ (LSD test) 
Table 8. Role of phosphorus foliar application on benefit cost ratio (BCR) of cotton 2015 \& 2016

\begin{tabular}{|c|c|c|c|c|c|c|c|c|}
\hline Years & Treatments & $\begin{array}{c}\text { Yield } \\
\left(\text { kg ha }^{-1}\right)\end{array}$ & $\begin{array}{c}\text { Value } \\
\left(\text { Rs ha }^{-1}\right)\end{array}$ & $\begin{array}{c}\text { Stick value } \\
\left(\text { Rs ha }^{-1}\right)\end{array}$ & $\begin{array}{c}\text { Gross value } \\
\left(\text { Rs ha }^{-1}\right)\end{array}$ & $\begin{array}{c}\text { Total cost } \\
\left(\text { Rs ha }^{-1}\right)\end{array}$ & $\begin{array}{c}\text { Net return } \\
\left(\text { Rs ha }^{-1}\right)\end{array}$ & BCR \\
\hline \multirow{4}{*}{$\mathbf{2 0 1 5}$} & Control $\left(\mathrm{P}_{0}\right)$ & 2116.9 & 148183 & 8000 & 156183 & 109950 & 46233 & 1.42 \\
\cline { 2 - 9 } & $50 \mathrm{~kg} \mathrm{ha}^{-1}\left(\mathrm{P}_{1}\right)$ & 2221.3 & 155491 & 8000 & 163491 & 110550 & 52941 & 1.47 \\
\cline { 2 - 9 } & $100 \mathrm{~kg} \mathrm{ha}^{-1}\left(\mathrm{P}_{2}\right)$ & 2221.4 & 155498 & 10000 & 165498 & 110950 & 54548 & 1.49 \\
\cline { 2 - 9 } & $150 \mathrm{~kg} \mathrm{ha}^{-1}\left(\mathrm{P}_{3}\right)$ & 2667.9 & 186753 & 10000 & 196753 & 112000 & 84753 & 1.75 \\
\hline \multirow{2}{*}{$\mathbf{2 0 1 6}$} & Control $\left(\mathrm{P}_{0}\right)$ & 1714.7 & 128602.5 & 8000 & 136602.5 & 106550 & 30052.5 & 1.28 \\
\cline { 2 - 9 } & $50 \mathrm{~kg} \mathrm{ha}^{-1}\left(\mathrm{P}_{1}\right)$ & 1778.0 & 133350 & 8000 & 141350 & 107400 & 33950 & 1.31 \\
\cline { 2 - 9 } & $100 \mathrm{~kg} \mathrm{ha}^{-1}\left(\mathrm{P}_{2}\right)$ & 1818.0 & 136350 & 10000 & 146350 & 109540 & 36810 & 1.33 \\
\cline { 2 - 10 } & $150 \mathrm{~kg} \mathrm{ha}^{-1}\left(\mathrm{P}_{3}\right)$ & 1814.7 & 136102.5 & 10000 & 146102.5 & 108950 & 37152.5 & 1.34 \\
\hline
\end{tabular}

$\mathrm{BCR}=$ benefit-cost ratio

\section{Conclusion}

Excess uses of nutrients over optimum level affects the economy of farmers. In respect to observe the concentration of fertilization that were supplemented to crops. Hence, it is very compulsory to improve endorsement programs that will mentioned the fixed fertilizer doses according to requirements of cotton crop. Present study concluded that the highest rate of phosphorus increase the cotton production and also increase the farmer's economy. Current study encourages the optimum use of phosphorus to avoid the excessive use of phosphors which leading higher input costs for farmer. Present study is also meaningful to improve the endorsement programs that will appropriately determine fertilizer supplements according to other crop needs.

\section{Authors' contributions}

Conceived and designed the experiments: $\mathrm{H}$ Ali, Performed the experiments: A Ahmad, Analyzed the data: R Ahmad, Contributed materials/ analysis/ tools: $\mathrm{H}$ Ali \& S Hussain, Wrote the paper: S Hussain \& A Ahmad.

\section{Acknowledgements}

The authors are very thankful to the Central Cotton Research Institute, Multan for providing the plant material and experimental site and Bahauddin Zakariya University, Multan for the financial support to carry out this work.

\section{References}

1. Gallagher JP, Grover CE, Rex K, Moran M \& Wendel JF (2017). A new species of cotton from Wake Atoll, Gossypium stephensii (Malvaceae). Syst Bot 42: 115-123.

2. Ali H, Ahmad A \& Hussain, S. (2020). The effect of exogenous phosphorous application on growth, yield, quality and net returns of upland cotton (Gossipium hirsutum L.). Appl Ecol Environ Res 18(1): 769-781.

3. Lenka NK \& Lal R (2012). Soil-related constraints to the carbon dioxide fertilization effect. Critic. Rev. Plant Sci 31(4): 342-357.

4. Vance CP, Uhde SC \& Allan DL (2003). Phosphorus acquisition and use: critical adaptations by plants for securing a nonrenewable resource. New Phytol 157: 423-447.

5. Campbell CD \& Sage RF (2006). Interactions between the effects of atmospheric $\mathrm{CO}_{2}$ content and $\mathrm{P}$ nutrition on photosynthesis in white lupin (Lupinus albus L.). Plant Cell Environ 29(5): 844-853.

6. Maqsood AG, Sabir MS, Ashraf, A \& Aziz R (2005). Effect of P-stress on growth, phosphorus uptake and 
utilization efficiency of different cotton cultivars. Pak J Agr Sci 42: 42-47.

7. Wang $\mathrm{X}$, Tang C, Guppy CN \& Sale PWG (2008). Phosphorus acquisition characteristics of cotton (Gossypium hirsutum L.), wheat (Triticum estivum L.) and white lupin (Lupinus albus L.) under phosphorus deficient conditions. Plant Soil 312: 117-128.

8. Ahmad Z, Ahmad Z, Gill MA, Qureshi RH, Rehman H \& Mahmood T (2001). Phosphorus nutrition of cotton cultivars under deficient and adequate levels in solution culture. Commun Soil Sci Plant Anal 32: 171-187.

9. Schwab GJ, Mullins GL \& Burmester CH (2000). Growth and nutrient uptake by cotton roots under field conditions. Commun. Soil Sci. Plant Anal. 31: 149164.

10. Amanullah, Asif M \& Nawab K (2010). Impacts of planting density and Pfertilizer source on the growth analysis of maize. Pak J Bot 42: 2349-2357.

11. Mahpara S, Shahnawaz M, Rehman K, Ahmad R \& Khan FU (2019). Nitrogen fertilization induced drought tolerance in sunflower: a review. Pure Appl Biol 8: 1675-1683.

12. Aamer M, Sabir MF \& Sadiq MA (2014). Exploiting genotypic variability among cotton cultivars for potassium use efficiency. Develop Country Studies 4: 7-12.

13. Bednarz CW \& Nichols RL (2005). Phenological and morphological components of cotton crop maturity. Crop Sci 45(4): 1497-1503.

14. Baloch MJ, Khan NU, Rajput MA, Jatoi WA, Gul S, Rind IH \& Veesar NF (2014). Yield related morphological measures of short duration cotton genotypes. J Anim Plant Sci 24: 11981211.

15. Gill KH, Sherazi SJA, Iqbal J, Ramzan M, Shaheen MH \& Ali ZS (2000). Soil fertility investigations on farmers' fields in Punjab, Soil Fertility Res. Inst., Depart. Agri., Govt. of Punjab, Lahore, Pakistan, pp. 133-135.
16. Malewar GU, Badole SB, Mali CV, Siddiqui MB \& Syed I (2000). Influence of flyash with and without FYM and fertilizer on physico-chemical properties of sunflower and cotton growing soils. Annal Agric Res 21: 187-191.

17. Saleem MF, Shakeel A, Bilal MF, Shahid MQ \& Anjum SA (2010). Effect of different phosphorus levels on earliness and yield of cotton cultivars. Soil Environ 29: 128-135.

18. Guidi L, Pallini M \& Soldatini GF (1994). Influence of phosphorus deficiency on photosynthesis in sunflower and soybean plants. AgrochiMica 38: 211-23.

19. Sawan ZM, Mahmoud MH \& Momtaz OA (1997). Influence of nitrogen fertilization and foliar application of plant growth retardants and zinc on quantitative and qualitative properties of Egyptian cotton (Gossypium barbadense L. var. Giza 75). J Agric Food Chem 45: 3331-6

20. Hunt, KH (1978). Oxford University Press, USA.

21. Ndor E, Agbede, OO \& Dauda SN (2010). Growth and yield response of cotton (Gossypium spp) to varying levels of nitrogen and phosphorus fertilization in Southern Guinea Savanna Zone, Nigeria. PAT 6: 119-125.

22. Ali H, Ahmad A \& Hussain S (2020). The effect of exogenous phosphorous application on growth, yield, quality and net returns of upland cotton (Gossipium Hirsutum L.). Appl Ecol Environ Res 18(1): 769-781.

23. Ali H, Muhammad D \& Abid SA (2005). Weed control practices in cotton (Gossypium hirsutum L.) planted on bed and furrow. Pak J Weed Sci Res 11: 4348.

24. Amouzou KA, Naab JB, Lamers JP, Borgemeister C, Becker M \& Vlek PL (2018). CROPGRO-Cotton model for determining climate change impacts on yield, water-and $\mathrm{N}$-use efficiencies of cotton in the Dry Savanna of West Africa. Agric Syst 165: 85-96. 